\title{
Feasibility of heart-rate monitoring to estimate total level and pattern of energy expenditure in a population-based epidemiological study: the Ely young cohort feasibility study 1994-5
}

\author{
BY NICHOLAS J. WAREHAM ${ }^{1}$, SUSIE J. HENNINGS ${ }^{1}$, ANDREW M. PRENTICE ${ }^{2}$ \\ AND NICHOLAS E. DAY ${ }^{1}$ \\ ${ }^{1}$ Department of Community Medicine, Institute of Public Health, University of Cambridge, \\ Cambridge, CB2 2SR \\ ${ }^{2}$ MRC Dunn Clinical Nutrition Centre, Hills Road, Cambridge, CB2 2DH
}

(Received 3 December 1996 - Revised 14 May 1997 - Accepted 9 June 1997)

\begin{abstract}
Increasing the precision of measurements of total energy expenditure in population-based epidemiological studies is important for accurately quantifying the relationship between this exposure and disease. Current questionnaire-based methods cannot accurately quantify total energy expenditure, although they may provide an estimate of the frequency of vigorous activities. Heart rate monitoring with individual calibration has been advocated as a method for assessing energy expenditure in field studies and has been compared with the 'gold standard' techniques of doublylabelled water and indirect calorimetry. However the method has previously only been used on small and selected populations. This study was, therefore, established to test the feasibility of using heart rate monitoring in a population-based study of adults. $A$ total of 167 individuals aged 30-40 years were randomly selected and underwent $4 \mathrm{~d}$ heart-rate monitoring. Only three individuals could not complete the protocol. The mean physical activity level (PAL) measured over $4 \mathrm{~d}$ was 1.89 (SD 0.40) in men and 1.76 ( $\mathrm{SD} \mathrm{0.31)}$ in women. There was no difference between mean PAL on weekend days compared with weekdays (mean paired difference $0.0008,95 \% \mathrm{CI}-0.06$ to +0.06 ). The estimate of mean PAL was not correlated with BMI, percentage body fat or the waist : hip ratio. It was, however, correlated with cardio-respiratory fitness as measured by $\mathrm{VO}_{2 \max }$ per $\mathrm{kg}$ (Spearman rank correlation coefficient 0.50 in men and 0.42 in women). The pattern of energy expenditure was assessed by calculating the percentage of daytime hours in which PAL was greater than five times basal energy expenditure. This measure was strongly correlated with the mean PAL in both men (Spearman correlation coefficient 0.77 ) and women (0.71). We conclude that heart-rate monitoring is a feasible method for assessing the pattern and total level of energy expenditure in medium-sized epidemiological studies. It may also prove useful as the reference technique for calibrating questionnaires to estimate energy expenditure in larger scale studies.
\end{abstract}

Energy expenditure: Physical activity: Exercise

Physical activity is a multi-dimensional behaviour (Caspersen, 1989) which cannot easily be condensed into a unitary measure. One of the failures of physical activity epidemiology has been to assume that a single instrument could adequately assess the various dimensions of activity, which include total energy expenditure, aerobic intensity, weight-bearing, flexibility and muscular strength. For some biological end-points such as obesity or obesity-related diseases like diabetes, measurement of the pattern and total level of energy expenditure is an important consideration. By contrast, for endpoints like osteoporosis, an assessment of participation in weight-bearing activity would be more relevant. Selecting an 
appropriate instrument to assess the dimension of physical activity that is of most interest to the particular endpoint of concern is an important prerequisite for the accurate description of the exposure-disease relationship.

The optimal method for assessing the total level and the pattern of energy expenditure in epidemiological studies is unknown. Questionnaires have been used in the majority of studies as they are easy to administer to large numbers of people and do not intrude on people's everyday activities, in contrast to other techniques such as diaries (Paffenbarger $e t$ al. 1993). Many such questionnaires were originally developed for use in studies aimed at investigating the link between physical activity and IHD (Haskell et al. 1992). Although certain questionnaires purport to estimate total energy expenditure, they concentrate on easily remembered and discrete episodes of moderate to vigorous activity. The 'validation' comparisons that have been made are usually against other questionnaires which almost certainly have correlated error, or against physiological variables such as fitness which are associated with total energy expenditure, but which are not direct measures of it (Jacobs et al. 1993; Miller et al. 1994; Richardson et al. 1995). Thus the validity of recall questionnaires to estimate energy expenditure has not been proven.

Direct measurement of energy expenditure is only appropriate for small studies. Doubly-labelled water (DLW) is expensive, while indirect calorimetry does not assess an individual in a free-living state. Indirect measurement of energy expenditure has therefore been investigated as a possible alternative. Although there have been improvements in commercially available movement sensors, these are still not very robust (Williams et al. 1989) and do not distinguish between load-bearing and non-load-bearing activity. The well-known relationship between energy expenditure and heart rate has led to interest in the potential for heart rate monitoring as a method for estimating energy expenditure. Early studies used accumulated or average pulse rate to predict energy expenditure (Payne et al. 1971; Dauncey \& James, 1979; Christensen et al. 1983). Subsequently some investigators moved to using minute-by-minute measurements of heart rate with linear prediction of energy expenditure. The use of a single calibration line was criticized because of the nonlinearity at low energy expenditure (Kalkwarf et al. 1989) leading to the development of methods using segmented linear or curvi-linear predictions. Overall, methods that use minute-by-minute measurement with individual rather than group calibration and which take account of the non-linearity at low energy expenditure have proved most accurate ( $\mathrm{Li}$ et al. 1993). The HRFlex method uses an individual calibration of the relationship between energy expenditure and heart rate on exertion together with measurement of resting energy expenditure and estimation of the heart rate at which the linear assumption does not hold, the 'FLEX' point. This FLEX heart rate is taken empirically as the mean of the highest pulse rate at rest and lowest on exercise (Ceesay et al. 1989). Below this point energy expenditure is assumed to be equal to rest, but above this it is estimated from the slope and intercept of the line between energy expenditure and exercise heart rate. The HRFlex method has been compared in validation studies with the 'gold standard' techniques of doubly-labelled water (Livingstone et al. 1990, 1992) and indirect calorimetry (Spurr et al. 1988). These comparisons suggest that the technique provides an accurate estimate of group energy expenditure but that there is imprecision at the individual level. In a comparative study by Ceesay et al. (1989) the mean error for estimating total energy expenditure by this technique was only $0.6 \%$.

As the heart rate monitoring method is relatively cheap, assesses individuals in a freeliving state and has the potential for providing information on the pattern as well as the total level of energy expenditure, it has been suggested as a method applicable to epidemiological studies whose primary interest is the relationship between energy 
expenditure and disease (Livingstone et al. 1992). However a number of other characteristics of the method remain to be investigated before its use can be recommended. Previous studies using heart rate monitoring have used selected populations (Schulz et al. 1989; Heini et al. 1991; Leonard et al. 1995; Racette et al. 1995; Spurr et al. 1996). Heartrate monitoring without individual calibration has been used in population-based studies in children (Armstrong et al. 1990). If the HRFlex technique were to be used in an epidemiological context, one would need to demonstrate that it was possible to recruit and study individuals from a population-based cohort. A technique that was only applicable to $60 \%$ of a cohort for example, would not be an appropriate measurement tool for estimating the population mean energy expenditure, as the $40 \%$ for whom measurement was not possible might be inherently different from those for whom it was. The technique of heartrate monitoring with individual calibration is data-intensive. It would therefore also be important to demonstrate that it was possible to process and analyse the large amount of information that would be produced in an epidemiological study. The aims of this study were therefore to examine the feasibility of using $4 \mathrm{~d}$ heart-rate monitoring with individual calibration as an epidemiological tool for estimating energy expenditure and to describe the pattern and total energy expenditure of a population of adults aged $30-40$ years (the Ely young cohort feasibility study 1994-5).

\section{SUBJECTS AND METHODS}

\section{Selection of the subjects}

An initial sampling frame was constructed from all individuals aged 30-40 years on 1 June 1994 who were registered with a large general practice in Ely, Cambridgeshire. One in ten random sampling was used to construct a sample of 365 individuals who were approached by letter co-signed by the subject's general practitioner and the study co-ordinator. This followed a local media campaign and a public meeting at which the results of a previous epidemiological study in Ely were discussed and this project was announced.

\section{Anthropometric measurements and questionnaires}

Volunteers were invited to attend the clinic at 08.30 hours and had fasted since 22.00 hours the previous evening as one of the biochemical tests that was undertaken was a standard $75 \mathrm{~g}$ oral glucose tolerance test. In keeping with World Health Organization recommendations (World Health Organization Study Group, 1985), volunteers were asked to abstain from smoking, or the use of either a nicotine patch or chewing gum on the morning of the test. Height and weight were measured in light clothing. Body circumferences were measured in duplicate using a metal tape. The waist circumference was measured at the mid-point between the lower costal margin and the level of the anterior superior iliac crest. Hip circumference was measured at the level of the greater trochanter. Body fat percentage was obtained using a standard impedance technique (Bodystat, Isle of Man). Self-reported physical activity was assessed using the Paffenbarger questionnaire (Paffenbarger $e$ t al. 1993; Wolf et al. 1994) with standard coding of specific activities using published energy costs for recreations and sports (Ainsworth et al. 1993). Ethical permission for the study was granted by the Cambridge District Ethics Committee. 


\section{Assessment of resting and exercise oxygen consumption-heart rate relationship}

The $\mathrm{O}_{2}$ consumption-heart rate relationship was assessed at rest with the subject lying prone and then seated, using an $\mathrm{O}_{2}$ analyser (PK Morgan Ltd, Rainham, Gillingham, Kent) calibrated daily using $100 \% \mathrm{~N}_{2}$ and fresh air as standard gases. Daily records of ambient room temperature and barometric pressure were recorded. Subjects were then asked to cycle on a cycle ergometer at several different workloads to provide the slope and the intercept of the line relating energy expenditure to heart rate. Each subject cycled at $50 \mathrm{rev} . / \mathrm{min}$ and the workload was progressively increased from $0 \mathrm{~W}$, through $37.5 \mathrm{~W}$, $75 \mathrm{~W}$ and $125 \mathrm{~W}$ in stages each lasting $5 \mathrm{~min}$. At each workload three separate readings of heart rate, minute volume and expired air $\mathrm{O}_{2}$ concentration were taken after allowing the subject $2 \mathrm{~min}$ to get used to the new level of work. The $125 \mathrm{~W}$ level was only undertaken if the heart rate had not reached 120 beats/min by the end of the 3 min at $75 \mathrm{~W}$. The $\mathrm{O}_{2}$ concentration in the expired air and minute volume data were used to calculate $\mathrm{O}_{2}$ consumption after correction for standard temperature and pressure. Energy expenditure $(\mathrm{kJ} / \mathrm{min})$ was calculated at each time point as $\mathrm{O}_{2}$ consumption $(\mathrm{ml} / \mathrm{min}) \times 20.35$ (Consolazio et al. 1963). Mean resting energy expenditure was taken as the average of the lying and sitting values. FLEX heart rate was calculated as the mean of the highest resting pulse rate and the lowest on exercise. Finally the slope and intercept of the least squares regression line of the exercise points were calculated. These calculations were computed using an SPSS syntax file (Statistical Package for the Social Sciences, SPSS Inc., Chicago, IL, USA). $\mathrm{VO}_{2 \max }$ was estimated from the linear regression as $\mathrm{O}_{2}$ consumption at maximal heart rate (220 minus age) and is expressed in the results per unit body weight (Harrison $e t$ al. 1980).

\section{Heart-rate information collection and data processing}

At the end of the morning testing session, the functions of the heart-rate monitor were explained to the volunteers who were asked to wear it continuously during the waking hours over the following $4 \mathrm{~d}$. They did not wear the monitor at night. A period of $4 \mathrm{~d}$ of monitoring was chosen for two practical reasons. First, the type of heart-rate monitor used in this study (Polar Sports Tester, Kempele, Finland) has a memory capacity of just over $34 \mathrm{~h}$. Therefore to cover the period of use, subjects were either given two watches or arrangements were made for the data to be downloaded after the second day. It would have been impractical for reasons of expense for subjects to have been given more heart-rate monitors. Second, pilot testing of the methods with volunteer subjects indicated that they became more aware of the chest belt after $4 \mathrm{~d}$ and that in some subjects skin irritation commenced at about this time especially in hot weather. As the intention of this study was to be as inclusive as possible, $4 \mathrm{~d}$ was selected so as to maximize information whilst minimizing drop-outs.

At the end of this $4 \mathrm{~d}$ period the heart-rate readings were directly downloaded into a computer via a serial interface. Because the data are transmitted between the chest piece and the watch receiver, the heart-rate monitor can sometimes be subject to interference. This is usually caused by being very close to certain types of motor such as hairdryers or vacuum cleaners. The interference is manifest as an apparent heart rate of about 220 beats/min. Occasionally loose contact on the chest wall means that readings of 0 beats/min are obtained for some minutes. We therefore wrote a Windows-based computer program which allowed each file to be examined for interference and zero readings. If any aberrant readings were observed, they were replaced by the average of the 
previous and subsequent values. If more than five aberrant readings occurred in succession, the data were not interpolated but rather the segment was removed. The program also removed the file headers and footers.

\section{Computation of energy expenditure}

The individual calibration data were used to predict minute-by-minute energy expenditure for each person. If heart rate was less than or equal to FLEX heart rate, then energy expenditure was assumed to be equal to mean resting energy expenditure. If heart rate was greater than FLEX heart rate, then energy expenditure was calculated from the linear prediction calculated previously. Again a Windows-based program was designed to allow batch processing of information. It was usual for subjects to miss a small segment of time both at the beginning and at the end of each day. For the purposes of calculating total energy expenditure, we assumed that this time was spent at rest. Sleeping energy expenditure was calculated as $95 \%$ of BMR (Goldberg et al. 1988) where this was derived from published prediction equations (James \& Schofield, 1990). In men in this age range, $\mathrm{BMR}=0.0485 \times$ weight $(\mathrm{kg})+3.67 \mathrm{MJ} / \mathrm{d}$ and in women, $\mathrm{BMR}=0.0364 \times$ weight $(\mathrm{kg})+3.47 \mathrm{MJ} / \mathrm{d}$. A physical activity level (PAL), which is the total energy expenditure : BMR ratio, was computed for each day and averaged over the $4 \mathrm{~d}$ period. The files of minute-by-minute energy expenditure during the waking hours were also analysed to calculate the individual pattern of energy expenditure. In this paper, two measures of the pattern of energy expenditure are reported. The first measure is the simple proportion of time that was spent with a heart rate less than FLEX. Second, the data are expressed as the proportion of time spent with energy expenditure at least five times greater than basal energy expenditure.

\section{RESULTS}

Forty-three of the sampling frame of 365 individuals aged $30-40$ years were ineligible for the study; thirty-two had moved away (two of them abroad), five were pregnant, three were hospitalized with long-term illnesses, one subject had insulin-dependent diabetes, one was detained at Her Majesty's Pleasure and one had died. A total of 167 subjects attended for screening but three individuals were unable to complete the heart-rate monitoring and individual calibration section. This was due to a severe learning difficulty in one individual and severe physical handicap in the other two. Therefore 164 or $50.8 \%$ of the eligible sample completed the protocol.

The anthropometric characteristics of this population are shown in Table 1. The overall estimate of PAL was 1.89 (SD 0.40) in men and 1.76 (SD 0.31) in women. As Table 2 shows there was no relationship in men or women between total energy expenditure and either BMI or percentage body fat as measured by impedance. In men, ANOVA showed that there was a positive linear trend between mean age and increasing quartiles of PAL $(P=0.02)$. In women there was a weakly significant linear trend in the opposite direction $(P=0.047)$ with women in the bottom quartile of PAL being older.

Table 3 shows the relationship between the overall level and pattern of energy expenditure. There was a strong negative linear trend between total energy expenditure and the proportion of the day-time hours spent with a pulse rate lower than FLEX heart rate $(P<0.001$ in men and women). Conversely there was a positive relationship between total energy expenditure and the proportion of time spent with energy expenditure greater than five times basal energy expenditure $(P<0.001$ in men and women). There was also a 
Table 1. Anthropometric features of the study population: the Ely young cohort feasibility study, 1994-5 (n 164)

\begin{tabular}{|c|c|c|c|c|}
\hline & \multicolumn{2}{|c|}{ Men ( $n 74)$} & \multicolumn{2}{|c|}{ Women $(n 90)$} \\
\hline & Mean & SD & Mean & SD \\
\hline Age (years) & $36 \cdot 2$ & 2.6 & $36 \cdot 3$ & 2.8 \\
\hline BMI $\left(\mathrm{kg} / \mathrm{m}^{2}\right)$ & 27.0 & 4.6 & 25.4 & 5.1 \\
\hline Body fat (\%) & $21 \cdot 2$ & $5 \cdot 5$ & $31 \cdot 7$ & 6.6 \\
\hline Waist : hip ratio & 0.92 & 0.07 & 0.78 & 0.05 \\
\hline
\end{tabular}

Table 2. Anthropometric features of subjects by physical activity level (PAL) quartile stratified by sex: the Ely young cohort feasibility study, 1994-5 (n 164)

(Mean values and standard deviations)

\begin{tabular}{|c|c|c|c|c|c|c|c|}
\hline \multirow[b]{2}{*}{ PAL quartile } & \multirow[b]{2}{*}{$n$} & \multicolumn{2}{|c|}{ Age (years) } & \multicolumn{2}{|c|}{ BMI $\left(\mathrm{kg} / \mathrm{m}^{2}\right)$} & \multicolumn{2}{|c|}{ Body fat (\%) } \\
\hline & & Mean & SD & Mean & SD & Mean & SD \\
\hline \multicolumn{8}{|l|}{ Men } \\
\hline$<1.615$ & 18 & $35 \cdot 2$ & $3 \cdot 1$ & $26 \cdot 1$ & $3 \cdot 2$ & $20 \cdot 1$ & 4.5 \\
\hline $1.615-1.825$ & 18 & $36 \cdot 4$ & 2.6 & 28.0 & 6.1 & 22.9 & $6 \cdot 5$ \\
\hline $1.825-2.03$ & 19 & 35.6 & 2.8 & 26.9 & 4.7 & $20 \cdot 5$ & $5 \cdot 6$ \\
\hline$>2.03$ & 19 & 37.7 & 2.4 & $26 \cdot 9$ & $4 \cdot 1$ & 20.8 & 4.7 \\
\hline \multicolumn{8}{|l|}{ Women } \\
\hline$<1.518$ & 22 & 37.0 & 2.4 & 25.4 & 5.8 & $32 \cdot 8$ & $7 \cdot 3$ \\
\hline $1.518-1.761$ & 22 & 36.5 & 2.8 & $25 \cdot 7$ & 5.8 & $32 \cdot 0$ & 7.1 \\
\hline $1.761-1.958$ & 23 & 35.9 & 2.5 & $24 \cdot 3$ & 3.8 & 29.9 & $5 \cdot 8$ \\
\hline$>1.958$ & 23 & 35.6 & $2 \cdot 7$ & $26 \cdot 3$ & $5 \cdot 1$ & 32.2 & 6.5 \\
\hline
\end{tabular}

Table 3. Relationship between indicators of pattern of energy expenditure and physical activity level (PAL) stratified by sex: the Ely young cohort feasibility study, 1994-5 (n 164)

(Values are means and standard deviations for $\mathrm{VO}_{2 \max }$ per $\mathrm{kg}$ and geometric means with $95 \%$ confidence intervals for other variables)

\begin{tabular}{|c|c|c|c|c|c|c|c|}
\hline \multirow{2}{*}{$\begin{array}{l}\text { PAL } \\
\text { quartile }\end{array}$} & \multirow[b]{2}{*}{$n$} & \multicolumn{2}{|c|}{$\begin{array}{c}\mathrm{VO}_{2 \max } \text { per } \mathrm{kg} \\
(\mathrm{ml} \mathrm{O} / \mathrm{kg} \text { per min) }\end{array}$} & \multicolumn{2}{|c|}{$\begin{array}{c}\% \text { daytime with } \\
\text { heart rate < FLEX HR }\end{array}$} & \multicolumn{2}{|c|}{$\begin{array}{c}\% \text { daytime } \\
\text { with } P A L \geq 5.0\end{array}$} \\
\hline & & Mean & SD & Mean & $95 \% \mathrm{CI}$ & Mean & $95 \% \mathrm{CI}$ \\
\hline \multicolumn{8}{|l|}{ Men } \\
\hline$<1.615$ & 18 & $32 \cdot 9$ & $5 \cdot 6$ & $52 \cdot 3$ & $43.8-62.4$ & 0.72 & $0.33-1.58$ \\
\hline $1.615-1.825$ & 18 & 34.9 & $8 \cdot 0$ & 41.7 & $37.0-47.1$ & $2 \cdot 15$ & $1 \cdot 32-3 \cdot 50$ \\
\hline $1 \cdot 825-2 \cdot 03$ & 19 & $38 \cdot 2$ & 6.7 & 21.5 & $15 \cdot 8-29 \cdot 2$ & 3.59 & $2 \cdot 50-5 \cdot 16$ \\
\hline$>2.03$ & 19 & 42.9 & 7.9 & 7.3 & $3 \cdot 5-15 \cdot 3$ & $13 \cdot 11$ & $8.60-19.99$ \\
\hline \multicolumn{8}{|l|}{ Women } \\
\hline$<1.518$ & 22 & $25 \cdot 1$ & 5.4 & $54 \cdot 3$ & $48 \cdot 8-60 \cdot 4$ & 0.36 & $0.25-0.53$ \\
\hline $1.518-1.761$ & 22 & 29.7 & $6 \cdot 7$ & $42 \cdot 1$ & $36 \cdot 4-48 \cdot 6$ & 1.05 & $0.70-1.58$ \\
\hline $1.761-1.958$ & 23 & 31.6 & $5 \cdot 3$ & 25.4 & $20 \cdot 9-30 \cdot 8$ & 1.86 & $1.29-2.69$ \\
\hline$>1.958$ & 23 & $32 \cdot 3$ & $6 \cdot 2$ & $13 \cdot 1$ & $8 \cdot 7-19 \cdot 9$ & 4.06 & $2 \cdot 64-6 \cdot 24$ \\
\hline
\end{tabular}

FLEX HR, 'flex' heart rate (the mean of the highest pulse rate at rest and lowest on exercise). 
Table 4. Spearman rank correlation between physical activity level (PAL) and anthropometric characteristics, $\mathrm{VO}_{2 \max }$ and indicators of pattern of energy expenditure in subjects from the Ely young cohort feasibility study, 1994-5 (n 164)

\begin{tabular}{lcc}
\hline \hline & Men & Women \\
\hline Age (years) & 0.22 & -0.20 \\
BMI ( $\left(\mathrm{kg} / \mathrm{m}^{2}\right)$ & 0.07 & 0.06 \\
Body fat $(\%)$ & -0.01 & -0.08 \\
Waist : hip ratio & 0.01 & 0.09 \\
VO $_{\text {2max }}$ per $\mathrm{kg}\left(\mathrm{ml} \mathrm{O}_{2} /\right.$ min per $\mathrm{kg}$ ) & $0.50^{* *}$ & $0.42^{* *}$ \\
Percentage day time with HR $<$ FLEX & $-0.72^{* *}$ & $-0.77^{* *}$ \\
Percentage day time with PAL $>5.0$ & $0.77^{* * *}$ & $0.71^{* * *}$ \\
\hline
\end{tabular}

HR, heart rate; FLEX, 'flex' heart rate (the mean of the highest pulse rate at rest and the lowest on exercise). ** $P<0.01, * * * P<0.001$.

strong relationship between total energy expenditure and cardio-respiratory fitness as assessed by the $\mathrm{VO}_{2 \max }$ per $\mathrm{kg}$. As the underlying data are continuous, we also examined the rank correlation with the estimate of energy expenditure (Table 4) stratified by sex. These data demonstrate the absence of a relationship between PAL and any of the measures of overall or regional adiposity measured in this cross-sectional study. The weak correlation between age and energy expenditure did not reach conventional statistical significance in either men or women. There was a significant positive relationship between total energy expenditure and cardio-respiratory fitness as assessed by the $\mathrm{VO}_{2 \max }$ per $\mathrm{kg}$. We also examined the continuous relationship between PAL and two measures of the pattern of physical activity. The correlation between PAL and the proportion of day-time hours spent with a pulse rate less than FLEX was strong, as was the correlation between PAL and the proportion of time spent with an energy expenditure greater than five times basal.

Physical activity was also quantified in this study using the Paffenbarger physical activity questionnaire. The estimate of energy expenditure from this questionnaire is expressed in MET (multiples of the resting metabolic rate; $1 \mathrm{MET} \simeq 4.18 \mathrm{~kJ}(1 \mathrm{kcal}) / \mathrm{kg}$ body weight per $\mathrm{h}$ ). Fig. 1 shows the geometric mean MET score by quartile of the heartrate monitor-estimated PAL. No simple relationship is apparent and the Spearman rank correlation between the underlying continuous data is minimal at 0.08 . The questionnaire assessment of energy expenditure was weakly correlated with cardio-respiratory fitness and more strongly with the proportion of time during the heart-rate monitoring period spent with energy expenditure greater than five times basal energy expenditure (Table 5).

The heart-rate monitor was worn for the $4 \mathrm{~d}$ following the clinic appointment and testing was performed on each day of the week except Sunday. Therefore subjects differed in the number of weekdays and weekend days that they wore the watch for. If there was an overall tendency for energy expenditure to be different on weekend days compared with week days, then this $4 \mathrm{~d}$ sampling strategy could introduce bias. To examine this possibility, we compared the estimates of PAL calculated for week days and weekend days for the ninety-seven subjects who had both. The overall mean PAL for weekdays was 1.8144 (SD 0.37) compared with 1.8135 (SD 0.384) for weekend days. The $t$ value for a paired $t$ test comparison was 0.03 (two-tail significance with 96 degrees of freedom $=0.98$ ). The mean paired difference between week days and weekend days was $0.0008(95 \% \mathrm{CI}=-0.060-0.061)$. Thus there is no evidence that the $4 \mathrm{~d}$ sampling 


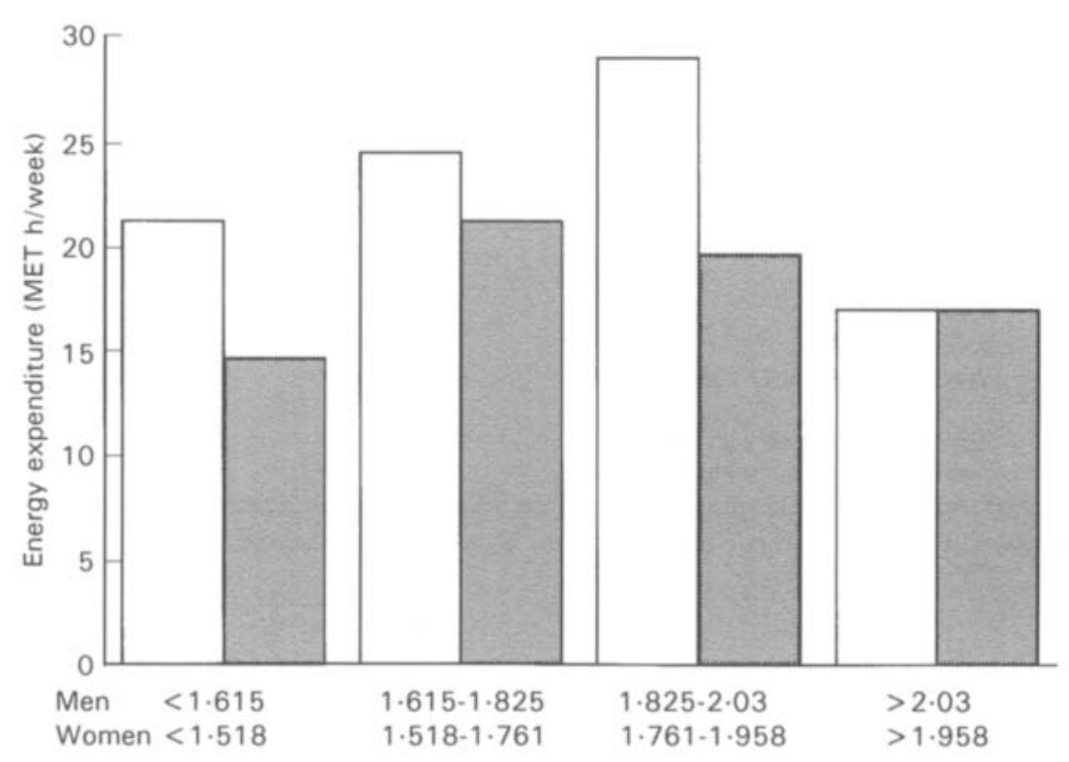

Fig. 1. Questionnaire-assessed energy expenditure by quartile of physical activity level estimated from heart-rate monitoring. Values are geometric means for men $(\square)$ and women (살) in the Ely young cohort feasibility study 1994-5 ( $n$ 164). MET, multiples of the resting metabolic rate; $1 \mathrm{MET} \simeq 4.18 \mathrm{~kJ}(1 \mathrm{kcal}) / \mathrm{kg}$ body weight per $\mathrm{h}$.

strategy would introduce bias. However it will introduce error or random noise as the estimates of week day and weekend energy expenditure are not perfectly correlated, see Fig. 2 (Pearson correlation coefficient 0.69).

\section{DISCUSSION}

The two main aims of this study were to examine the feasibility of using heart-rate monitoring with individual calibration as an epidemiological tool for estimating energy expenditure and to use the technique to describe the pattern and total energy expenditure in a population-based study. The study has successfully demonstrated that it is possible to use heart-rate monitoring in relatively large samples and that the systems for managing the data can be developed. An important feature of an epidemiological instrument is that it should be widely applicable. Only three of the volunteers who participated in this study were

Table 5. Spearman rank correlation between total energy expenditure assessed by Paffenbarger questionnaire and estimates from heart-rate monitoring (HRM): the Ely young cohort feasibility study, 1994-5 (n 164).

\begin{tabular}{lc}
\hline \hline & $\begin{array}{c}\text { Questionnaire estimate of total energy } \\
\text { expenditure (MET } \dagger \text { h/week) }\end{array}$ \\
\hline $\mathrm{HRM}$ estimated PAL & 0.08 \\
$\mathrm{VO}_{2 \max }$ per $\mathrm{kg}\left(\mathrm{ml} \mathrm{O}_{2} / \min\right.$ per $\left.\mathrm{kg}\right)$ & $0.15^{*}$ \\
Percentage day time with PAL $>5.0$ & $0.25^{* *}$ \\
\hline
\end{tabular}

PAL, physical activity level.

$* P<0.05,{ }^{* *} P<0.01$.

$\dagger$ Multiples of the resting metabolic rate; 1 MET $\simeq 4.18 \mathrm{~kJ}(1 \mathrm{kcal}) / \mathrm{kg}$ body weight per $\mathrm{h}$. 


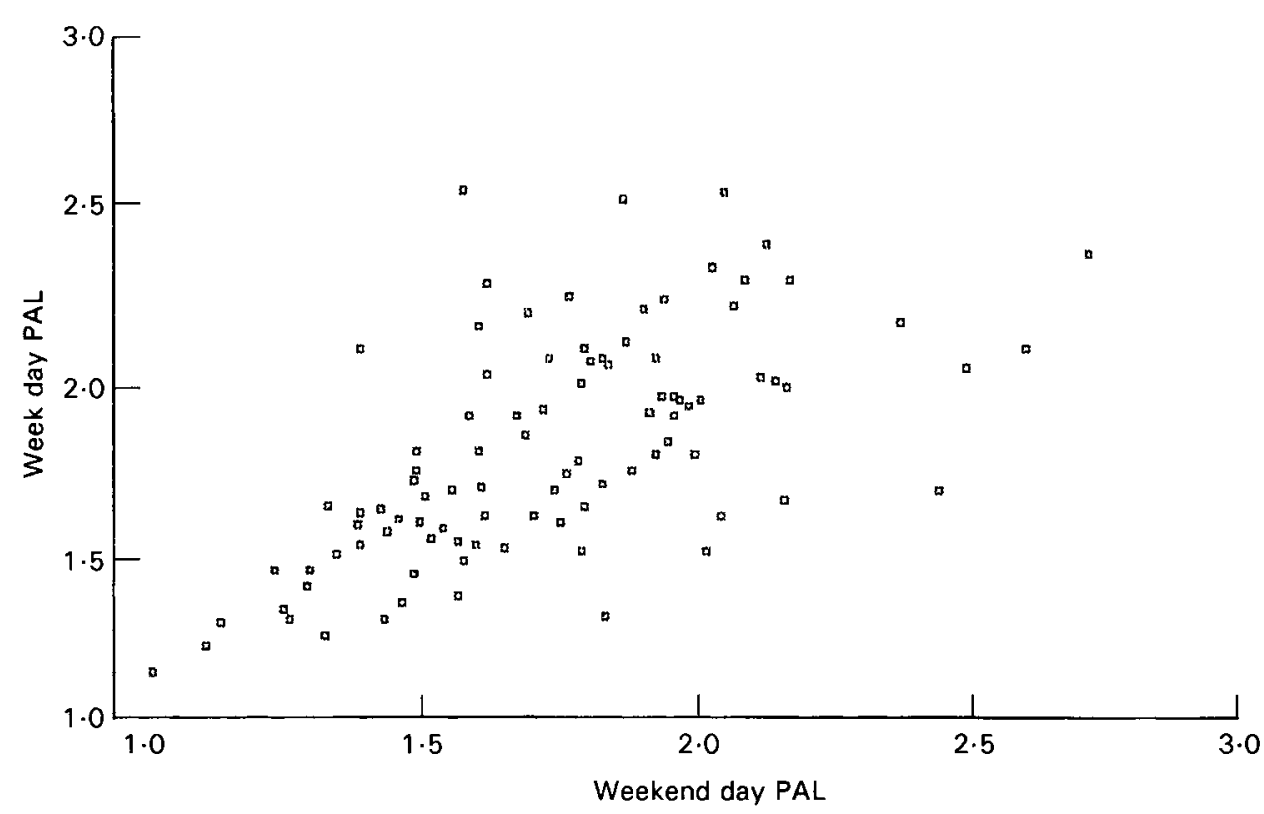

Fig. 2. Comparison of physical activity levels (PAL) measured on week days with those measured on weekend days in subjects from the Ely young cohort feasibility study, 1994-5 ( $n$ 97).

unable to complete the protocol and therefore the method was appropriate for over $98 \%$ of the cohort. However, the volunteers were young and we would expect that the method would be less suitable if a higher proportion of older subjects were included. The main exclusions would be co-incident disease which precluded the exercise component of the calibration and the use of high doses of beta blockers which would greatly reduce the tachycardia of physical activity. An overall response rate of $50 \%$ was achieved for this study. The modest response rate is a function of all epidemiological studies of this type in young adults and is not a consequence of the heart-rate monitoring method itself. Although generalization of these results must be treated with caution, it is encouraging that the estimates of total energy expenditure in this population are comparable with those from a recent meta-analysis of DLW measurements (Black et al. 1996). In that study the estimate of mean PAL by DLW in the age range $30-39$ years was 1.77 (SD 0.31 ) in thirty-six men, and 1.68 (SD 0.25) in seventy-six women. There was no significant difference between these mean estimates for PAL and those estimated by heart-rate monitoring in the present study. The study participants in the Ely study are likely to be more representative of the general population, as many of the studies which formed the basis of the meta-analysis selected volunteers without regard to a broader population of interest. However, major confounding by obesity is unlikely as there was no significant difference in mean BMI between the two studies. The mean BMI for men aged 30-39 years in the DLW metaanalysis was $26.8(\mathrm{SD} 8.8) \mathrm{kg} / \mathrm{m}^{2}$ and that for women $25.2(\mathrm{SD} 4.9) \mathrm{kg} / \mathrm{m}^{2}$.

Although we have demonstrated the feasibility of heart-rate monitoring in this study, its use in very-large-scale epidemiological studies may be limited by the time that is needed for the individual calibration and the collection of the data. The technique clearly has a role for medium-sized longitudinal cohorts and for estimating energy expenditure in repeat cross-sectional studies. Although temporal trend data are available for energy intake, 
trends in energy expenditure are assumed rather than measured. The availability of a technique which could serve this purpose would be an important contribution to the study of the aetiology of obesity (Prentice \& Jebb, 1995). The ability to make repeated measurement of energy expenditure in individuals would also allow heart-rate monitoring to act as the reference technique for estimating usual energy expenditure against which a questionnaire could be calibrated. It is also possible to describe the relationship between a single estimate of energy expenditure and the habitual or usual level. If a single measurement of $4 \mathrm{~d}$ energy expenditure is to be used as an estimate of usual energy expenditure, then the relationship between them must be quantified. This would allow the measured association between the single estimate of energy expenditure and outcome to be adjusted for the regression dilution bias (MacMahon et al. 1990). This question is considered in a second paper from this study in which a sub-group of twenty-two individuals underwent $4 \mathrm{~d}$ heart-rate monitoring on four occasions over 1 year.

In the present study, the PAL was associated with cardiorespiratory fitness. However the degree of association was such that it would be possible to demonstrate independent effects provided the sample size were sufficiently large. If fitness is seen as the end-product of conditioning exercise (above a genetically determined level), then theoretically it would be possible to have high overall energy expenditure without being particularly fit if the majority of the energy expenditure was at an intermediate non-conditioning level. The ability to separate these different effects is important if one considers extrapolating from epidemiological evidence to public health action. Thus studies that are able to separate the independent effects of fitness and total energy expenditure would be of value. The correlation between the individual mean physical activity level and the proportion of time spent with energy expenditure greater than five times basal, by contrast, was much greater at 0.77 in men and 0.71 in women. This implies that the amount of vigorous physical activity is an important determinant of the overall mean. This degree of correlation may make it difficult to separate the effects of the pattern from the overall level.

In the pilot work for this study, we identified that individuals became more aware of the monitor after $4 \mathrm{~d}$. For this reason, and because of the limitation imposed by the limited monitor memory length which was then available, we elected to ask volunteers to wear the monitor only for $4 \mathrm{~d}$. Thus some individuals were sampled for only weekdays, while others who came for testing on a Saturday wore the monitor for two weekend days and two weekdays. The practical constraints of using this technique in an epidemiological study meant that it was impossible for us to have enough monitors to allow all subjects to be studied on weekend and week days. This might introduce bias into this study if overall there was a tendency for energy expenditure to be greater at the weekend than during the week. The comparison of weekend with week day energy expenditure in this study suggests that there is no difference in the population mean. In other words there are as many people who are active during the week but less active at the weekend as vice versa. Therefore the strategy that we have adopted for epidemiological studies in which no regard is taken to the days of the week during which heart-rate monitoring is undertaken does not introduce bias, but will of course introduce error or noise. However, the strength of the correlation between week day and weekend energy expenditure suggests that this error will not be very large.

As a comparison in this study we also used the Paffenbarger questionnaire which has been widely used in studies of physical activity and disease. The three main components of the questionnaire are estimated energy expenditure from self-reported averaged past year participation in walking, climbing stairs and sports and recreations. A priori, we postulated that this questionnaire would assess the frequency of vigorous activity with greater 
precision than total energy expenditure because vigorous activities are recalled more easily than less vigorous activity. The correlation between the Paffenbarger estimate of total energy expenditure and that from heart-rate monitoring was very low, but there was a significant positive correlation with the proportion of time spent with energy expenditure above five times basal. This comparison is between a single estimate of $4 \mathrm{~d}$ energy expenditure and self-reported activity over a much longer time period and therefore one might not expect a high degree of correlation. However, the difference in the strength of the relationship between the questionnaire estimate and the total level and pattern of energy expenditure from heart-rate monitoring, suggests that the questionnaire may only be an indicator of the frequency of vigorous activities. Improved knowledge of the precise exposure that is being estimated by this and other questionnaires would be an important contribution, as it would allow appropriate design of intervention strategies.

This study was funded by the Anglia and Oxford Regional Health Authority. We are grateful to the staff and patients of the St Mary's Street Surgery, Ely and to H. Shannasy, S. Curran, J. Mitchell, J. Lipscombe, P. Murgatroyd and Dr M. Hennings for their help with the fieldwork and analysis of this study.

\section{REFERENCES}

Ainsworth, B. E., Haskell, W. L., Leon, A. S., Jacobs, D. R., Montoye, H. J., Sallis, J. F. \& Paffenbarger, R. S. (1993). Compendium of physical activities: classification of energy costs of human physical activities. Medicine and Science in Sports and Exercise 25, 71-80.

Armstrong, N., Balding, J., Gentle, P.\& Kirby, B. (1990). Patterns of physical activity among 11 to 16 year old British children. British Medical Journal 301, 203-205.

Black, A. E., Coward, W. A., Cole, T. J. \& Prentice, A. M. (1996). Human energy expenditure in affluent societies: an analysis of 574 doubly-labelled water measurements. European Journal of Clinical Nutrition $\mathbf{5 0}$, $72-92$.

Caspersen, C. J. (1989). Physical activity epidemiology: concepts, methods, and applications to exercise science. Exercise Sports Science Reviews 17, 423-473.

Ceesay, S. M., Prentice, A. M., Day, K. C., Murgatroyd, P. R., Goldberg, G. R., Scott, W. \& Spurr, G. B. (1989). The use of heart rate monitoring in the estimation of energy expenditure: a validation study using indirect whole-body calorimetry. British Journal of Nutrition 61, 175-186.

Christensen, C. C., Frey, H. M. M., Foenstelien, E., Aadland, E. \& Refsum, H. E. (1983). A critical evaluation of energy expenditure estimates based on individual $\mathrm{O}_{2}$ consumption/heart rate curves and average daily heart rate. American Journal of Clinical Nutrition 37, 468-472.

Consolazio, C. F., Johnson, R. E. \& Pecora, L. J. (1963). Physiological Measurements of Metabolic Functions in Man. New York: McGraw Hill.

Dauncey, M. J. \& James, W. P. T. (1979). Assessment of the heart rate method for determining energy expenditure in man, using a whole body calorimeter. British Journal of Nutrition 42, 1-13.

Goldberg, G. R., Prentice, A. M., Davies, H. L. \& Murgatroyd, P. R. (1988). Overnight and basal metabolic rates in men and women. European Journal of Clinical Nutrition 42, 137-144.

Harrison, M. H., Bruce, D. L., Brown, G. A. \& Cochrane, L. A. (1980). A comparison of some indirect methods of predicting maximal oxygen uptake. Aviation Space and Environmental Medicine 51, 1128.

Haskell, W. L., Leon, A. S., Caspersen, C. J., Froelicher, V. F., Hagberg, J. M., Harlan, W., Holloszy, J. O., Regensteiner, J. G., Thompson, P. D., Wasburn, R. A. \& Wilson, P. W. F. (1992). Cardiovascular benefits and assessment of physical activity and physical fitness in adults. Medicine and Science in Sports and Exercise 24, S201-S220.

Heini, A., Schutz, Y., Diaz, E., Prentice, A. M., Whitehead, R. G. \& Jequier, E. (1991). Free-living energy expenditure measured by two independent techniques in pregnant and non-pregnant Gambian women. American Journal of Physiology 261, E9-E17.

Jacobs, D. R., Ainsworth, B. E., Hartman, T. J. \& Leon, A. S. (1993). A simultaneous evaluation of 10 commonly used physical activity questionnaires. Medicine and Science in Sports and Exercise 25, 81-91.

James, W. P. T. \& Schofield, E. C. (1990). Human Energy Requirements. Oxford: Oxford Medical Publications.

Kalkwarf, H. J., Haas, J. D., Belko, A. Z., Roach, R. C. \& Roe, D. A. (1989). Accuracy of heart-rate monitoring and activity diaries for estimating energy expenditure. American Joumal of Clinical Nutrition 49, 37-43. 
Leonard, W. R., Katzmarzyk, P. T., Stephen, M. A. \& Ross, A. G. P. (1995). Comparison of the heart ratemonitoring and factorial methods: assessment of energy expenditure in highland and coastal Ecuadoreans. American Joumal of Clinical Nutrition 61, 1146-1152.

Li, R., Deurenberg, P. \& Hautvast, J. G. A. J. (1993). A critical evaluation of heart rate monitoring to assess energy expenditure in individuals. American Journal of Clinical Nutrition 58, 602-607.

Livingstone, M. B. E., Coward, W. A., Prentice, A. M., Davies, P. S. W., Strain, J. J., McKenna, P. G., Mahoney, C. A., White, J. A., Stewart, C. M. \& Kerr, M-J. J. (1992). Daily energy expenditure in free-living children: comparison of heart-rate monitoring with the doubly labeled water $\left({ }^{2} \mathrm{H}_{2}{ }^{18} \mathrm{O}\right)$ method. American Journal of Clinical Nutrition 56, 343-352.

Livingstone, M. B. E., Prentice, A. M., Coward, W. A., Ceesay, S. M., Strain, J. J., McKenna, P. G., Nevin, G. B., Barker, M. E. \& Hickey, R. J. (1990). Simultaneous measurement of free-living energy expenditure by the doubly labeled water method and heart-rate monitoring. American Journal of Clinical Nutrition 52, 59-65.

MacMahon, S., Peto, R., Cutler, J., Collins, R., Sorlie, P., Neaton, J., Abbott, R., Godwin, J., Dyer, A. \& Stamler, J. (1990). Blood pressure, stroke and coronary heart disease: Part 1, prolonged differences in blood pressure: prospective observational studies corrected for the regression dilution bias. Lancet 335, 765-774.

Miller, D. J., Freedson, P. S. \& Kline, G. M. (1994). Comparison of activity levels using the Caltrac accelerometer and five questionnaires. Medicine and Science in Sports and Exercise 26, 376-382.

Paffenbarger, R. S., Blair, S. N., Lee, I.-M. \& Hyde, R. T. (1993). Measurement of physical activity to assess health effects in free-living populations. Medicine and Science in Sports and Exercise 25, 60-70.

Payne, P. R., Wheeler, E. F. \& Salvosa, C. B. (1971). Prediction of daily energy expenditure from average pulse rate. American Journal of Clinical Nutrition 24, 1164-1170.

Prentice, A. M. \& Jebb, S. A. (1995). Obesity in Britain: gluttony or sloth? British Medical Journal 311, $437-439$.

Racette, S. B., Schoeller, D. A. \& Kushner, R. F. (1995). Comparison of heart rate and physical activity recall with doubly labelled water in obese women. Medicine and Science in Sports and Exercise 27, $126-133$.

Richardson, M. T., Ainsworth, B. E., Wu, H.-C. \& Jacobs, D. R. (1995). Ability of the Atherosclerosis risk in communities (ARIC)/Baeke questionnaire to assess leisure-time physical activity. International Journal of Epidemiology 24, 685-693.

Schulz, S., Westerterp, K. R. \& Bruck, K. (1989). Comparison of energy expenditure by the doubly labeled water technique with energy intake, heart rate and activity recording in man. American Journal of Clinical Nutrition 49, 1146-1154.

Spurr, G. B., Dufour, D. L. \& Reina, J. C. (1996). Energy expenditure of urban Colombian women: a comparison of patterns and total daily expenditure by the heart rate and factorial methods. American Journal of Clinical Nutrition 63, 870-878

Spurr, G. B., Prentice, A. M., Murgatroyd, P. R., Goldberg, G. R., Reina, J. C. \& Christman, N. T. (1988). Energy expenditure from minute-by-minute heart-rate recording: comparison with indirect calorimetry. American Joumal of Clinical Nutrition 48, 552-559.

Williams, E., Klesges, R. C., Hanson, C. L. \& Eck, L. H. (1989). A prospective study of the reliability and convergent validity of three physical activity measures in a field research trial. Journal of Clinical Epidemiology 42, 1161-1170.

World Health Organization Study Group (1985). Diabetes Mellitus. Technical Report Series no. 727. Geneva: WHO.

Wolf, A. M., Hunter, D. J., Colditz, G. A., Manson, J. E., Stampfer, M. J., Corsano, K. A., Rosner, B., Kriska, A. \& Willett, W. C. (1994). Reproducibility and validity of a self-administered physical activity questionnaire. International Journal of Epidemiology 23, 991-999. 